\title{
Comment on "Head-on collision of electron acoustic solitary waves in a plasma with nonextensive hot electrons"
}

\author{
Frank Verheest ${ }^{1,2}$ \\ frank. verheest@ugent. be
}

\begin{abstract}
In a recent paper "Head-on collision of electron acoustic solitary waves in a plasma with nonextensive hot electrons" [Astrophys. Space Sci. 338, 271-278 (2012)] Eslami, Mottaghizadeh and Pakzad deal with the problem of the head-on collisions between two weakly nonlinear electronacoustic solitary waves. Unfortunately, their treatment is deficient and leads to erroneous conclusions.
\end{abstract}

Subject headings: plasmas - waves

In a recent paper, Eslami, Mottaghizadeh and Pakzad est orders gives from (EMP.3) or (11) that (2012) deal with the problem of the head-on collisions between two weakly nonlinear electronacoustic solitary waves in a two-electron plasma with hot nonextensive and cold components, in the presence of a neutralizing ion background. Unfortunately, their treatment is deficient and leads to erroneous conclusions.

For clarity in what follows, equations from the paper by Eslami. Mottaghizadeh and Pakzad (2012) will be denoted as (EMP.1) and higher, and equations in this Comment as (1) and higher. Let us start the discussion from the Poisson equation (EMP.3), which is repeated here for ease of exposition:

$\frac{\partial^{2} \phi}{\partial x^{2}}-\frac{1}{\alpha} n_{c}-[1+(q-1) \phi]^{\frac{q+1}{2 q-2}}+\left(1+\frac{1}{\alpha}\right)=0$.

The cold electron density $n_{c}$ has to be determined from the relevant fluid equations (EMP.1) and (EMP.2). Using their expansions (EMP.6) to low-

\footnotetext{
${ }^{1}$ Sterrenkundig Observatorium, Universiteit Gent, Krijgslaan 281, B-9000 Gent, Belgium

${ }^{2}$ School of Chemistry \& Physics, University of KwaZuluNatal, Private Bag X54001, Durban 4000, South Africa
}

This part is straightforward, since terms with derivatives only occur to third or higher order. The order of the expansion in $\varepsilon$ is denoted by superscripts, in their notation, and their expansions (EMP.6) start with terms in $\varepsilon$ outside equilibrium.

In (EMP.10) a separable form is proposed for $\phi^{1}$,

$$
\phi^{1}=\phi_{1}^{1}(\xi, \tau)+\phi_{2}^{1}(\eta, \tau),
$$

which gives (EMP.11) or

$$
n^{1}=-\alpha \frac{q+1}{2}\left[\phi_{1}^{1}(\xi, \tau)+\phi_{2}^{1}(\eta, \tau)\right]
$$

and thus fulfils (2). However, Eslami. Mottaghizadeh and Pakzad (2012) claim that $n^{2}$ and $\phi^{2}$ have separability properties analogous to the first order ones, cfr. (EMP.13) and (EMP.14), hence

$$
\begin{aligned}
\phi^{2} & =\phi_{1}^{2}(\xi, \tau)+\phi_{2}^{2}(\eta, \tau), \\
n^{2} & =-\alpha \frac{q+1}{2}\left[\phi_{1}^{2}(\xi, \tau)+\phi_{2}^{2}(\eta, \tau)\right] .
\end{aligned}
$$

It is immediately clear that this cannot hold, since there would remain from (3) that

$$
\alpha \frac{(q+1)(q-3)}{8}\left[\phi_{1}^{1}(\xi, \tau)+\phi_{2}^{1}(\eta, \tau)\right]^{2}=0,
$$


and except for $q=3$ one would have to conclude that $\phi_{1}^{1}(\xi, \tau)=0$ and $\phi_{2}^{1}(\eta, \tau)=0$, wiping out all first order terms.

The problem is worse, because the equations of continuity (EMP.1) and of motion (EMP.2) also contain nonlinear contributions to second order. One can try to eliminate e.g. $u^{2}$ to arrive at another relation between $n^{2}$ and $\phi^{2}$, but disentangling the information is bedevilled by the fact that derivatives with respect to $\xi$ and $\eta$ occur together, preventing an immediate integration.

A way forward would be to propose a general decomposition

$$
\phi^{2}=\phi_{1}^{2}(\xi, \tau)+\phi_{2}^{2}(\eta, \tau)+\phi_{3}^{2}(\xi, \eta, \tau) .
$$

This includes a mixed term $\phi_{3}^{2}(\xi, \eta, \tau)$, which cannot be separated into parts not depending either on $\eta$ or on $\xi$, as these would be in $\phi_{1}^{2}(\xi, \tau)$ or $\phi_{2}^{2}(\eta, \tau)$, respectively. Using a similar decomposition for $n^{2}$ (and also for $u^{2}$, but let us concentrate on the densities and the electrostatic potential) allows to determine first from (EMP.1) and (EMP.2) that

$$
\begin{aligned}
& n_{1}^{2}=-\alpha \frac{q+1}{2} \phi_{1}^{2}(\xi, \tau)+\frac{3}{8}\left[\alpha(q+1) \phi_{1}^{1}(\xi, \tau)\right]^{2}, \\
& n_{2}^{2}=-\alpha \frac{q+1}{2} \phi_{2}^{2}(\eta, \tau)+\frac{3}{8}\left[\alpha(q+1) \phi_{2}^{1}(\eta, \tau)\right]^{2} .
\end{aligned}
$$

When this is substituted into (3) the terms in $n_{1}^{2}(\xi, \tau), n_{2}^{2}(\eta, \tau), \phi_{1}^{2}(\xi, \tau)$ and $\phi_{2}^{2}(\eta, \tau)$ cancel, because of the linear dispersion properties, so that for the terms only in $(\xi, \tau)$ or $(\eta, \tau)$ there remains that

$$
\begin{aligned}
& {[3 \alpha(q+1)+3-q]\left[\phi_{1}^{1}(\xi, \tau)\right]^{2}=0,} \\
& {[3 \alpha(q+1)+3-q]\left[\phi_{2}^{1}(\eta, \tau)\right]^{2}=0,}
\end{aligned}
$$

after having divided out common nonzero factors. In addition, when one combines the mixed contributions and eliminates $n_{3}^{2}(\xi, \eta, \tau)$ and $u_{3}^{2}(\xi, \eta, \tau)$, there is a differential equation for $\phi_{3}^{2}(\xi, \eta, \tau)$ to fulfil,

$$
\begin{aligned}
\frac{\partial^{2}}{\partial \xi \partial \eta} \phi_{3}^{2}(\xi, \eta, \tau)=\frac{1}{8} & {\left[(3-q)\left(\frac{\partial}{\partial \eta}-\frac{\partial}{\partial \xi}\right)^{2}\right.} \\
& \left.-\alpha(q+1)\left(\frac{\partial}{\partial \xi}+\frac{\partial}{\partial \eta}\right)^{2}\right] \times \\
\times & \phi_{1}^{1}(\xi, \tau) \phi_{2}^{1}(\eta, \tau) .
\end{aligned}
$$

Now the choice is clear.

A first and generic possibility is that $q$ does not annul the coefficients in (12) and (13), but then all first order variables vanish and (14) indicates that $\phi_{3}^{2}(\xi, \eta, \tau)=0$. Hence, the second order is indeed separable as claimed by Eslami, Mottaghizadeh and Pakzad (2012), but there is no first order left, $\phi_{1}^{1}(\xi, \tau)=0$ and $\phi_{2}^{1}(\eta, \tau)=0$, and from here on the remainder of the paper is automatically null and void.

The other choice is that $q$ is special, $q=(3)(1+$ $\alpha) /(1-3 \alpha)$, so that the first order variables remain in the loop, but for the second order quantities besides (10) and (11), one has to find a solution for $\phi_{3}^{2}(\xi, \eta, \tau)$, which is far from trivial but certainly nonzero, as (14) becomes

$$
\begin{aligned}
\frac{\partial^{2}}{\partial \xi \partial \eta} \phi_{3}^{2}(\xi, \eta, \tau)= & \frac{2 \alpha}{3 \alpha-1}\left(\frac{\partial^{2}}{\partial \xi^{2}}+\frac{\partial^{2}}{\partial \eta^{2}}-\frac{\partial^{2}}{\partial \xi \partial \eta}\right) \times \\
& \times \phi_{1}^{1}(\xi, \tau) \phi_{2}^{1}(\eta, \tau) .
\end{aligned}
$$

About this part of the discussion the authors are completely silent, and now the second order variables certainly are not given by (EMP.13)(EMP.15), so that also here the remainder of the paper presents no valid information.

Analogous criticisms invalidate the results in an earlier paper by the same authors (Eslami, Mottaghizadeh and Pakzad 2011), dealing with ion rather than electron acoustic modes, with obvious notational differences but having a similar structure.

Some of the papers in the literature start the expansion with terms in $\varepsilon^{2}$ (outside equilibrium), thereby implicitly assuming (apparently without checking!) that the model is simple enough so that the coefficients corresponding to those in (12) and (13) never vanish. Then the terms in $\varepsilon^{3}$ of the expansion do not contribute, and to the next order the relevant $\mathrm{KdV}$ equations and phase shifts are obtained.

This is certainly the case when a simple plasma model is considered with cold ions and Boltzmann electrons, without additional species Demiray 2007), but not immediately for many other models treated in the literature.

However, the plasma model investigated by Eslami. Mottaghizadeh and Pakzad (2012) is rich enough to admit critical values for the parameters, and so they were right to start their expansions with terms in $\varepsilon$, but did not work that out 
as it should have been, with the unfortunate consequence that their paper is incorrect and incomplete, either way.

There are other, but far less important, blemishes in the paper by Eslami, Mottaghizadeh and Pakzad (2012). One is that in (EMP.8) a factor $-\alpha$ is missing in front of the last two terms, as can immediately be seen by referring to the original equation of motion (EMP.2).

Another is that $\lambda$ is used in two different meanings, once in (EMP.12) and (EMP.15) where it really should be the velocity $c$ mentioned in the stretching (EMP.5), whereas the other $\lambda$, defined towards the bottom of the left hand column on page 2 , is essentially $1 / c^{2}$. Furthermore, there is an evident typo in the nonlinear term in (EMP.18).

It is interesting to remark that the value of $q$ which annuls the coefficient in (12) and (13) also annuls the coefficient $A$ of the nonlinear term in the KdV equations (EMP.17) and (EMP.18). Given the way the nonlinearities work, this should not come as a surprise.

To conclude, the paper by Eslami. Mottaghizadeh and Pakzad

(2012) is marred by an erroneous algebra (for generic $q+1>0$ ) or by a deficient discussion (when $q$ takes on a critical value), leaving the paper without validity.

\section{REFERENCES}

Demiray, H.: Interactions of nonlinear ionacoustic waves in a collisionless plasma. J. Comput. Appl. Math. 206, 826-831 (2007).

Eslami, P., Mottaghizadeh, M., Pakzad, H. R.: Head-on collision of ion-acoustic solitary waves in a plasma with a $q$-nonextensive electron velocity distribution. Physica Scripta 84, 015504 (2011).

Eslami, P., Mottaghizadeh, M., Pakzad, H. R.: Head-on collision of electron acoustic solitary waves in a plasma with nonextensive hot electrons. Astrophys. Space Sci. 338, 271-278 (2012).

This 2-column preprint was prepared with the AAS LATEX macros v5.2. 